\title{
STUDY ON APPLICATIONS OF WEB MINING TO DIGITAL LIBRARY
}

Baoji Wang, Ruiqing Xu, Jinnuo Zhu, Qingsheng Luo, Guoming Cheng, Wenli Yang, Zhihua Xin, Luyan Wang, Qingshui Liu

China Agricultural Univeristy Library, 17 Tsinghua East Road, Beijing,100083, P.R.China

Abstract: Based on the discussion of the technology properties of Data Mining and Web Mining, the types of Web mining were analyzed, the applications of Web mining to digital library and the Agricultural Engineering Digital Library (AEDL) based upon modern network, information and web mining technology were introduced.

Key words: Digital library, Data Mining, Web Mining, Information Service

\section{INTRODUCTION}

Digital library is a data information system, which contains abundant and diverse digital information resources, and depends on the support of modern technologies. It meets the need of users effectively by adopting modern new advanced technology and organizing resource orderly. At present, the information content of digital library comprises large number of digital library collection, various databases, full-text Web resource link and a mass of information in Internet. These considerable data must be organized, analyzed and mined to find out the valuable information, which the user need really. This is also the problem that the Web data mining technology wants to resolve. Web data mining technology is adopted and applied in the whole process of information discovery and supply of information in digital library to provide optimized information service and meet the individual need of user. Therefore, the Web data mining has expensive application space in several aspects as the optimum building of digital library 
information, information automated handling, advancing of information service quality and extension of obligation. And Web data mining will play an important role in the development of digital library to automation, networking and intelligence.

\subsection{Web Mining and Data Mining (DM)}

\subsubsection{Data Mining}

Data mining is a process of extracting connotative and unknown but useful information and knowledge from the vast, incomplete, noisy, fuzzy and stochastic data. DM brings the database technology into a more advanced phase. It cannot only inquiry the departed data, but also can find out the potential relationship between the old-time data, thereby DM can promote the information transfer. Briefly, DM is an advanced handing process that screens out connotative, believable, novel and effective information from a mass of data.

DM syncretizes statistic methods, computer technology and artificial intelligence technology, and extracts mode, amend, relationship, abnormity, regularization from the vast databank and statistic important structure and event.

DM was achieved mainly by followed methods: classification, regression, clustering, summarization, change and deviation detection and association rules.

\subsubsection{Web Mining}

Web data mining is the application of data mining technology to net information handing. It is the process that extracts the useful information or knowledge from web resource and distills the unknown mode, which contained in Web resource and has potential application value. Considered as integrated technique, Web data mining technology relates to multi-fields as Web technology, artificial intelligence, computer linguistics, informatics and statistics.

\subsubsection{Distinguish betw Web Content Mining een Web mining and data mining}

Web mining grows out of data mining, compared to data mining, Web mining has own characteristic as followed: firstly, the objects of Web mining 
are large number of heterogeneity distributing Web documents, and each data source is isomerous. The mining work towards the databank with Web as middle-piece, as well as towards the data of log and user information in Web server still belongs to traditional data mining. However, these types of data mining are considered as Web mining in most literatures presently. Secondly, Web is a logic diagram consists of document node and hyperlink. Therefore, the mode obtained from Web mining may be about Web content, and also can be about Web structure. Thirdly, Web is unstructured, semistructure and lack of semantic that machine can understand, and the objects of traditional data mining are limited in structural data of database. Thereby, many data mining technologies cannot apply to Web mining, although Web document need pretreatment.

\section{CHARACTERISTICS OF WEB DATA MINING}

Web is a distributed information system based upon hypertext. At present, Web involves many information service fields, such as news, advertisement, consumption information, finance management, education, government, electronic business. These information services provide abundant resource to data mining. The characteristics of Web data mining were listed as followed:

(1) Most data of Web is HTML format, and the information about some subject is distributed desultorily in multi-directories of Web site. In this way, a strong search engine is needed, by searching keywords to locate the hypertext. And an intelligentized system is also needed to understand the data described by natural language. It was difficult to understand these data completely, due to the immature natural language understanding technology presently. At the same time, there are redundancy, inconsistency and even contradictory between data source.

(2) Web seems to be too large to effective data storage and data mining. At present, web data is calculated by millions megabyte, and still increases rapidly, and has following two characteristics:

(1) Data source has very strong dynamic. Internet is a system that updates and changes momently. Therefore, it need use some technologies of data storage to save the renewed date of Web.

(2) The date has diversity. After filtrated, Web data has numeric data (integer, real), Boolean data, classified data, property metadata and proper Web data (URL and Email address et al). The new data type must generate new characteristic, and intrinsic mining method need to be improved and expended. 
(3) The aim of user is fuzzy. Data mining user based on Internet always has a passing acquaintance with the mining object and has not a specific aim. So the data mining system need to has some intelligence and learning mechanism, tail the interests of user continuously, and expatiate on the results of mining distinctly and detailedly.

Based upon the above characteristics, Web data mining is a challenge subject, refers Web access mode, Web structure and regulation, and dynamic Web content research.

\section{TYPES OF WEB DATA MINING}

The tasks of Web mining are diverse due to the complexity of Web information. Based on the different mining objects, Web data mining can be divided into following types: Web Content Mining, Web Structure Mining and Web Usage Mining. The classified structure is shown in Fig.1.

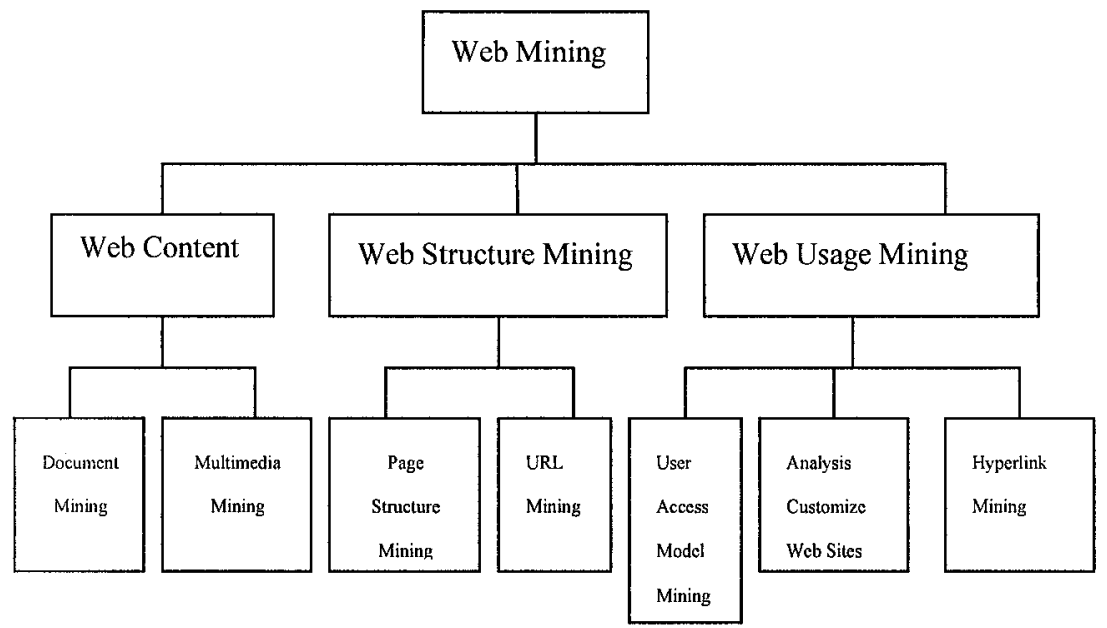

Figure 1. Classification of Web Mining

\subsection{Web Content Mining}

Web content mining is a process that obtains potential and valuable knowledge or mode from the file content and information of Web. Web content mining is divided into Web text mining and Web multimedia mining, and the mining objects are Web text information and Web multimedia information, respectively. 
(1) The mining towards text document (including Txt, PostScrip, PDF, HTML and so on) is considered as text mining. Web text mining can summarize, classify, cluster and relation associate analysis the content of vast document aggregate in Web, as well as can use Web document to analysis the trend.

The general treatment process of Web text data mining is described in Fig.2. Most document data of Web is html document and lack of structure characteristic as databank planar table. Thereby, characteristic information of html document aggregate that expressed as VSM generally must be extracted firstly. In this way, the document eigenvector has stupendous dimension, therefore, characteristic aggregate must be curtailed. Then, knowledge mode faced to specific application purpose is extracted by the method of machine learning. Finally, the gained knowledge mode is evaluated.

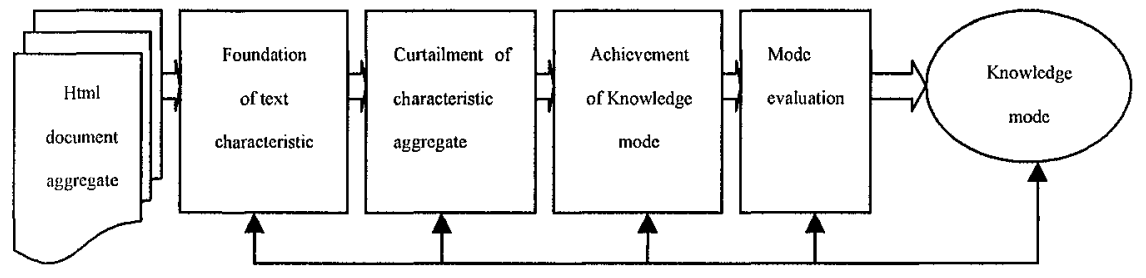

Figure 2. The general process of Web text data mining

(2) To mine the multimedia document (Image, Audio, Video) is called multimedia mining. The process of multimedia information mining is that pre-treats the audio-data, video data and image in Web firstly, and then mines the potential and significant information and mode by applying mining technology.

\subsection{Web Structure Mining}

Web structure mining is to infer the knowledge out of Web content by using organization structure and hyperlink relationship of Web page and institution information of Web document (Title, Heading, Anchor sign), it comprises hyperlink mining, inter structure mining and HTML mining. The hyperlink between documents reflects some affiliation of documents, such as inclusion, affiliation, correlativity, citation. Web page is analyzed by HTML tree logic structure to attain the characteristic of inter structure. Moreover, the HTML of Web page may reflect the type of page or directory structure relationship between pages. Web structure mining can summarize the structure of website and web page, and generates information of comparability and relationship between websites. It also can sort and cluster the web pages, and find the association between the web pages, then, judge 
the importance of pages and find the authoritative site of the correlative subject. Page Rank method and Key Page or Scripture method are the common methods. The new position of shifted Web Page is found by constructing heuristic regulation about Web Page HTML. The mining based on link can resolve the problem of network wander, and it can improve the using efficiency of Page by optimizing and recomposing dynamically the hyperlink.

\subsection{Web Usage Mining}

To mine the log left by the users when they accessed Web server is Web usage mining, and it is also called Web log mining. Web log mining discovers the browse mode of access site of users by analyzing log file of Web serve to provide multifarious information which profits the improvement of Web sites to the site administrators, such as the navigation function of Web sites and system design of Web application. Accordingly, Web usage mining benefits users, improves the performance of Web server and increases individual service.

The data treated by Web log mining contains: server log (access log, citation $\log$ and agency $\log$ ), choice data as the structure of Web sites and login number. The basic processes of Web mining shown in Fig.3 consist of pretreatment, transaction recognition, mining arithmetic actualization and mode analysis.

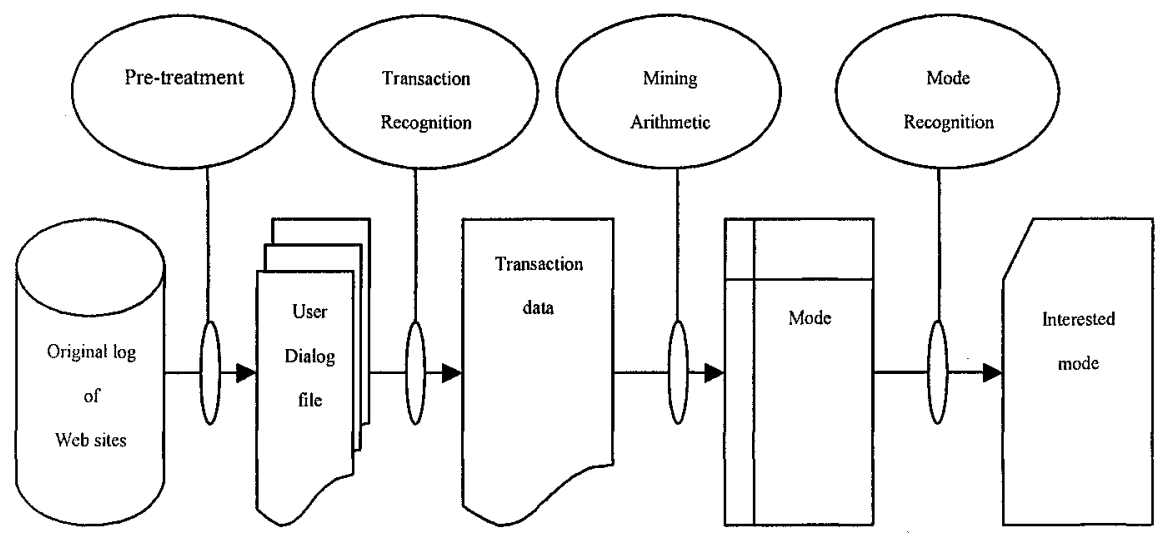

Figure 3 The basic process of Web log mining 


\section{APPLICATIONS OF WEB DATA MINING TO DIGITAL LIBRARY}

\subsection{Applications of Web data mining to information discovery}

The applications of Web data mining to information discovery comprise Web data exploitation techniques, multilingual information discovery and interdisciplinary coordinated searches. Web data exploitation technology is developed due to the difficulty of gaining the information from Internet, the realization process consists of information collection, document identification, document classification and others. Network intelligence and intelligent information catcher are the two realization manners. Multilingual information discovery is a research project developed for the geographical and lingual obstruction of globalization information. And the study involves several aspects as followed: multilingual electronic document getting, integrated machine translate and multilingual information searches system. Interdisciplinary coordinated search can send concurrent request to several even tens of databanks, and links with OPAC, interlibrary loan, document submission and electronic source. It provides uniform searches interface to users and returns to uniform results after interdisciplinary querying.

\subsection{Applications of Web data mining to service improvement}

User information and user access log are the two types of user access data that can be recorded when the user accesses digital library with browser. The user information includes user name, user access IP address, profession, age and hobby and so on. User name is inputted by user when the user enters into the library. The profession, age and hobby and other information of user are filled in when the user register the library. The user access IP address can attain via program. User access record embodies the click record of classified browse item and keywords and mode of search. The user access record also can obtain from program. To analysis the user access data, it can help the digital library understand well the user requirements in following aspects:

(1) To guide the collection of digital library information source. The default of information source can be discovered by analyzing the data of fail demand of user in server and integrating the assemble arithmetic to direct the collection of information source, then it makes the information source systems of digital library rational. The data of online investigation, message 
note, reading guide slip and others in digital library system can be transformed into normal structure database by collecting, clearing up and reconstructing. The data mining methods can be used to discover the interest mode of user and even pre-detect the interest vicissitude of user colony, adjust the direction of library collection, and do a collection plan of information source ahead.

(2) To improve the design of library site structure. The information demand and action rule of user can be found by using Web mining to provide reference to network site structure optimization. Sequentially, it makes the source configuration rational and the speech that user finds out the demanded information quicker. The measures that improve the design of library site structure include following aspects:

Investigating the user address in high access rate resource;

Making mirror image in concentrated region of user (obtaining by analyzing IP address);

Mining, catching and confirming the frequent browser and access path by using path analysis mode, adjusting the site structure;

Appending advertisement or reading guide slip in suited place.

\subsection{Applications of Web data mining to information supply}

One of the applications of Web data mining to information supply is to individual service, supplying actively the user with integrated and relative intact information or knowledge aggregate to meet the special demand of user. There are three types of individual service: (1) according to the specific user demand, providing customizing Web page and information channel or information item to user, putting the query agent service in practice; (2) according to specific subject, to direct literature source or supply literature full text, and to bring the individual literature information service into effect; (3) according to specific subject, offering relative intact project knowledge and carrying individual decision-making support service. These processes as followed must be experienced to actualize the individual service: Constructing individual user dynamic demand model; Searching and mining correlative information toward specific demand; According to specific subject, filtering, processing and assembling the gained information to integrate the relative intact information aggregate, and sending it to the user or service agent online or offline to realize information support. According to specific subject, syncretizing and activating the relative intact information aggregate to generate new project knowledge, and sending it to the user online or offline to realize decision-making support. The mining of Web 
usage $\log$ is the best method to realize this process in Web data mining technology.

Another application is information intelligent pusher, on the basis of the keyword that the user inputs, it can identify and forecast the interest and fancy of user, thereby, it pushes relative knowledge and up to date information to user in time. The form of push has channel push, mail push, web page push or special push.

The third application is interactive service, it emphasizes the building of uniform information supply platform, and let the different group that has different type of information and technology actualize information sharing. Based on these, the new information is generated by clustering and calculating.

\section{TO BUILD AN AGRICULTURAL ENGINEERING DIGITAL LIBRARY}

In order to supply agricultural engineering information more efficiently, accurately and completely for agricultural technician, we are making use of modern network, information and web mining technology to build an Agricultural Engineering Digital Library (AEDL).

AEDL has seven subsystems as followed:

Data source collection system, it has several functions as information collection on the web, existing electronic document format transition and metadata system transition.

Data source processing system, it is used to complete the digital processing and indexing classification of traditional source.

Database issuance and search system: to actualize various search service (full text retrieval, heterogeneous database uniform retrieval and distributed retrieval), and to realize subscription push, content issuance, full text transmission and Compact Disc publication.

Data source management system, it can actualize user purview management, databank maintenance, statistic and meterage.

Data reference consulting system: including such modules, such as FAQ consultation, MAIL consultation, QQ character interactive consultation, audio and video frequency real time reference and BBS.

User education system on the web, it consists of multimedia schoolteaching learning system, multimedia homework and examination system, online reference and tutorship system and courseware make tools, and can realize interactive distance learning based on network real time interactive technology. 
Personal digital library system, which actualizes individual personal source storage, management and collection, and the personal resource bank is divided into document data section, work and study section and entertainment section.

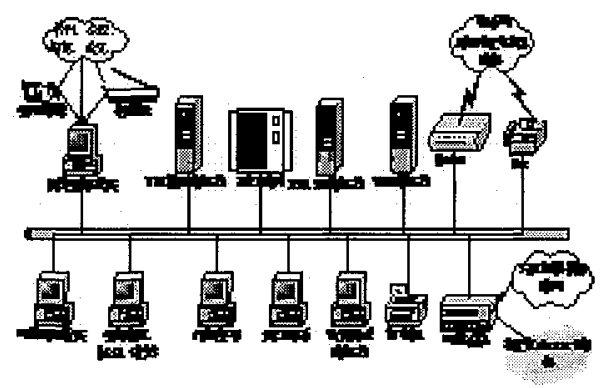

Figure 4 AEDL system structure

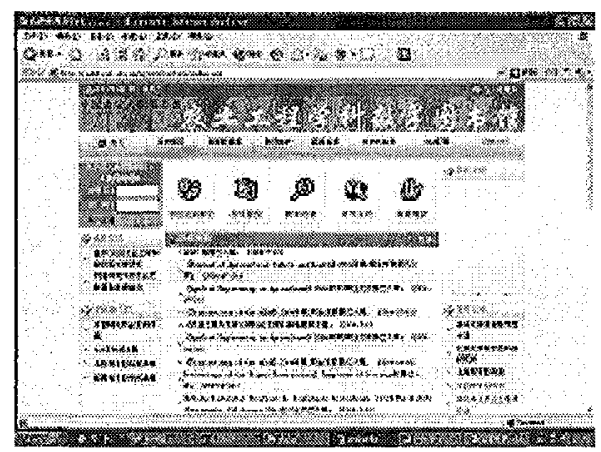

Figure 5 The Home Page of AEDL

\section{CONCLUSIONS}

Presently, there are many fruits in various aspects of knowledge discovery research. The study of Web data mining in digital library not only can utilize the exiting artificial intelligence theory well and can develop knowledge discovery research deeply, but also can obtain study fruits with comprehensive application foreground. Thereby, it has great value of research and application. Thus it can be seen that comprehensive integrated applications of knowledge discovery technology can resolve many problems of digital library currently, and it will accelerate the development of digital 
library radically by integrating the knowledge discovery applications into digital library system.

\section{REFERENCE}

[1]Arms W Y. Digital Libraries. Massachusetts, London: The MIT Press Cambridge, 2000

[2]Wang Yan. The Application of Data Mining in Digital Library. Information Science, 2003(2)

[3]Zhou Tao, Li Jun. The Research of Web Data Mining Technology, Journal of HanZhoug Teachers College (Natural Science), June, 2004

[4]Liu Wenqing, Bao Juejie. Research on Web-based Data Mining Technology and Its Application, Journal of Chongqing Three Gorges University, 2004(3)

[5]Bao Junjie. Research on Web Data Mining, Journal of Chongqing College of Education, May, 2004

[6]Wang Baoji. Study and Practice on Agricultural Engineering Digital Library Based on TPI, New Technology of Library and Information Service, 2003(5)

[7]He Shaozhuo. A few words about Data Mining and its Application to Library, Library World, sep., 2004 